\title{
Comment on "Electrochemical study of tetraalkylammonium tetrafluoroborate electrolytes in combination with microporous and mesoporous carbon monoliths" [Electrochimica Acta 268 (2018) 121-130]
}

Teresa A. Centeno

Instituto Nacional del Carbón (INCAR-CSIC). Francisco Pintado Fe 26. 33011 Oviedo

(Spain)

Keywords: Carbon electrode, Surface area, Tetraalkylammonium tetrafluoroborate electrolyte, Supercapacitor

The understanding of the electrical energy storage by carbons in supercapacitors (SC) has notably progressed over the last years. Among other key parameters, it has been found that more important than the specific surface area of the carbon used as active material in the electrodes is the surface involved in ions electrosorption [1]. The use of independent and complementary techniques has pointed out that the surface area accessible to the ions of the different electrolytes usually differ considerably from the total surface area of carbons, depending on the ions dimensions and the pore size distribution of the electrodes [1-4].

Whereas the total surface of carbons is determined successfully by combining several methods [2,5-7], the assessment of the extent of the electrochemical double-layer is an actual challenge. This difficulty coupled with uncertainties regarding the size of ions when confined into the porous carbon network have driven the need for systematic studies to get coherent conclusions. 
The large number of approaches currently available for determining the textural features of carbons [5,7] causes that they are frequently chosen on demand without being aware of their weaknesses. This often leads to inaccurate conclusions on carbon-based SC.

In a recent paper published in this journal, Moreno-Fernandez et al. [8] estimate the limiting dimension of the ions $\mathrm{BF}_{4}^{-}, \mathrm{Et}_{4} \mathrm{~N}^{+}, \mathrm{Pr}_{4} \mathrm{~N}^{+}$and $\mathrm{Bu}_{4} \mathrm{~N}^{+}$from the size of the pores capable of hosting them. The conclusions derive from "the comparison of the ratios of the specific capacitance of the anion and the three cations with the ratios of the surface areas estimated for pores with sizes larger than certain values". From the best agreement of both rations, critical dimensions of $0.56-0.58 \mathrm{~nm}$ for $\mathrm{BF}_{4}^{-}, 0.63-0.65 \mathrm{~nm}$ for $\mathrm{Et}_{4} \mathrm{~N}^{+}, 0.75-0.76 \mathrm{~nm}$ for $\mathrm{Pr}_{4} \mathrm{~N}^{+}$and $0.9-1.0$ $\mathrm{nm}$ for $\mathrm{Bu}_{4} \mathrm{~N}^{+}$are claimed. The surface-capacitance for each ion is subsequently calculated from the corresponding gravimetric capacitance and the surface in pores wider than the size estimated for each ion.

The study is based on two carbon monoliths, MesoCM and MicroCM, which are tested as SC electrodes in $1 \mathrm{M}$ solutions of the salts $\mathrm{Et}_{4} \mathrm{NBF}_{4}, \mathrm{Pr}_{4} \mathrm{NBF}_{4}$ and $\mathrm{Bu}_{4} \mathrm{NBF}_{4}$ in acetonitrile. The study of the electrodes porosity involves $\mathrm{N}_{2}$ adsorption/desorption at $77 \mathrm{~K}$ and immersion calorimetry but, surprisingly, a uniform protocol is not followed for both materials. The assessment of the micropore size distribution (PSD) and the cumulative surface area of MesoCM electrodes essentially rests on the analysis of the $\mathrm{N}_{2}$ isotherm by the Non-Local Density Functional Theory (NLDFT). It results puzzling that this approach is completely avoided for the textural characterization of MicroCM. Whereas its specific surface area is evaluated from the $\mathrm{N}_{2}$ isotherm analysis by the Dubinin-Radushkevich equation and the comparison plot, the PSD of MicroCM electrodes is derived exclusively by immersion calorimetry using 5 liquids such as dichloromethane $(0.33 \mathrm{~nm})$, benzene $(0.41 \mathrm{~nm})$, carbon tetrachloride $(0.63 \mathrm{~nm})$, cyclododeca1,5,9-triene CDDT $(0.76 \mathrm{~nm})$ and tri-2,4-xylylphosphate TXP $(1.50 \mathrm{~nm})$ as molecular probes.

Immersion calorimetry has proven to be a powerful tool for a refined textural characterization of porous carbons [10]. As it provides information on the accessibility of the 
micropore system and on the surface chemistry, it is also being highly useful for the study of carbons in SC $[3,4,11]$. Nevertheless, despite its potential, Stoeckli and Centeno [12] have already drawn attention to the limitations of this technique when used alone for calculating the surface areas of microporous solids.

The strength of immersion calorimetry in carbon-based supercapacitors is to be able to estimate the surface accessible to an ion by using a liquid with a molecular size conforming to the ion dimension. Thus, recent studies showed that immersion calorimetry using carbon tetrachloride $(0.63 \mathrm{~nm})$ or 2,5-norbornadiene $(0.65 \mathrm{~nm})$ as molecular probes matching the critical dimension of the cation $\mathrm{Et}_{4} \mathrm{~N}^{+}(0.68 \mathrm{~nm})$ provided direct information on the surface of microporous carbons which is available for the organic electrolyte $\mathrm{Et}_{4} \mathrm{NBF}_{4}$ in acetonitrile [2,3]. However, the straightforward application of a similar approach suggested by Moreno-Fernandez et al. [8] for microCM with tetraalkylammonium tetrafluoroborate electrolytes presents important weaknesses.

The histogram displayed in Fig. 1a illustrates the PSD found previously for MicroCM [9] and the corresponding cumulative surface area as a function of the micropore size is shown in Fig. 1b. At glance, there is a clear disagreement with the profile recently reported by MorenoFernandez et al. (Fig. 1b). The authors indicate that their calculations are based on previous immersion calorimetry data [9] but details are not provided.

The comparison of the surface areas summarized in Table 1 confirms a remarkable divergence, their values being $35 \%$ higher than those derived directly by immersion calorimetry [9]. Indeed, such a difference does not modify the ratios of the surface areas estimated for pores with sizes larger than certain values (Table 2) but it does notably affect the calculation of the surface-capacitance C/S (Table 3). If one uses consistently the surface values determined experimentally by immersion calorimetry, $\mathrm{C} / \mathrm{S}$ increases up to around $0.11 \mathrm{~F} \mathrm{~m}^{-2}$ for the ions in the presence of MicroCM. This value is significantly different with respect to $0.081 \pm 0.002$ and $0.078 \pm 0.003 \mathrm{~F} \mathrm{~m}^{-2}$ outlined by Moreno-Fernandez et al. [8] for MicroCM and MesoCM. 
Secondly, an important question is about the accuracy of the information obtained from immersion calorimetry based on such a limited number of molecular probes. For example, the immersion of MicroCM in CDDT and TXP indicates $513 \mathrm{~m}^{2} \mathrm{~g}^{-1}$ [9] located in pores between 0.76 and $1.5 \mathrm{~nm}\left(698 \mathrm{~m}^{2} \mathrm{~g}^{-1}\right.$ reported by Moreno-Fernandez et al.) but it is not possible to discriminate how they are distributed in the range. This surface value results from a gross estimate by

$$
S_{0.76-1.5}\left(\mathrm{~m}^{2} \mathrm{~g}^{-1}\right)=\frac{2000 W_{0.76-1.5}\left(\mathrm{~cm}^{3} \mathrm{~g}^{-1}\right)}{L_{a v}(\mathrm{~nm})}
$$

being $\mathrm{W}_{0.76-1.5}$ the micropore volume between 0.76 and $1.5 \mathrm{~nm}$ (derived from immersion calorimetry) and assuming slit-shaped micropores with an average size $\mathrm{L}_{\mathrm{av}}$ of $1.13 \mathrm{~nm}$. In fact, the lack of liquid probes with intermediate molecular dimensions prevents an accurate evaluation of the surface in such a wide range and, consequently, the surface values of $S_{>} 0.9$ and $S_{>} 1.0$ obtained by simple interpolation are also unreliable.

The validity of the approach outlined by Moreno-Fernandez et al. is definitely challenged by the DFT-analysis of gas adsorption data of MicroCM. As recently claimed, $\mathrm{N}_{2}$ adsorption at $77 \mathrm{~K}$ is not sufficiently accurate to probe materials with ultramicropores $(<0.8 \mathrm{~nm})$ and $\mathrm{CO}_{2}$ adsorption at $273 \mathrm{~K}$ has to be implemented for a better assessment of the porosity characteristics of carbons for supercapacitors [1].

Fig. 1 contains the pore size distribution (a) and cumulative surface area (b) of MicroCM from the analysis of $\mathrm{CO}_{2}$ adsorption by NLDFT complementing that of the $\mathrm{N}_{2}$ isotherm by the advanced Quenched Solid State Functional Theory (QSDFT). The latter takes into account surface geometrical inhomogeneity. The divergence observed in the region of narrow micropores in comparison to that from immersion calorimetry likely reflects the presence of constrictions at the entrance of the pores which hinder the access of $\mathrm{CCl}_{4}$. Nevertheless, the most noticeable difference is in the distribution of pores larger than $0.76 \mathrm{~nm}$. For pores above $0.9 \mathrm{~nm}$, Moreno- 
Fernandez et al. report a surface area as high as $560 \mathrm{~m}^{2} \mathrm{~g}^{-1}$ versus $70 \mathrm{~m}^{2} \mathrm{~g}^{-1}$ obtained by the DFT method. Similarly, their rough estimation of $473 \mathrm{~m}^{2} \mathrm{~g}^{-1}$ in pores above $1.0 \mathrm{~nm}$ is challenged by gas adsorption which reveals $S_{>1.0}$ of only $40 \mathrm{~m}^{2} \mathrm{~g}^{-1}$ (Table 1 ).

The clear contradiction between the ratios of surface obtained by the present DFT-analysis and the ratios of the specific capacitance of the anion $\mathrm{BF}_{4}{ }^{-}$and the three cations $\mathrm{Et}_{4} \mathrm{~N}^{+}, \mathrm{Pr}_{4} \mathrm{~N}^{+}$and $\mathrm{Bu}_{4} \mathrm{~N}^{+}$(Table 2) suggests inconsistencies in the approach outlined by Moreno-Fernandez et al. The different data quoted in Table 1 also have an impact on the assessment of the capacitance normalized to the accessible surface and Table 3 compiles strongly diverging results of surfacecapacitance for the different ions, depending on the choice of the surface area of MicroCM.

Finally, the fact that the sets of C/S values based on the DFT-characterization differ between MicroCM and MesoCM further stresses the weakness of this approach.

A more comprehensive characterization of the MesoCM monolith is also highly required to verify the experimental results obtained exclusively by HS-2D-NLDFT analysis of $\mathrm{N}_{2}$ adsorption. This has to be complemented by the use of $\mathrm{CO}_{2}$ adsorption at $273 \mathrm{~K}$ due to the significant presence of pores around $0.6 \mathrm{~nm}$ [8] as well as immersion calorimetry to eliminate possible bottle-neck pores. This would allow reaching coherent insights on the basis of uniform protocols for both carbon electrodes.

\section{References}

[1] B. Lobato, L. Suárez, L. Guardia, T.A. Centeno, Capacitance and surface of carbons in supercapacitors, Carbon 122 (2017) 434-445.

[2] T.A. Centeno, O. Sereda, F. Stoeckli, Capacitance in carbon pores of 0.7 to $15 \mathrm{~nm}$ : a regular pattern, Phys. Chem. Chem. Phys. 13 (2011) 12403-12406.

[3] A. García-Gómez, G. Moreno-Fernández, B. Lobato, T.A. Centeno, Constant capacitance in nanopores of carbon monoliths, Phys. Chem. Chem. Phys. 17 (2015) 15687-15690. 
[4] S. Pohlmann, B. Lobato, T.A. Centeno, Andrea Balducci, The influence of pore size and surface area of activated carbons on the performance of ionic liquid based supercapacitors, Phys.Chem. Chem. Phys. 15 (2013) 17287-17294.

[5] T.A. Centeno, F. Stoeckli, The assessment of surface areas in porous carbons by two modelindependent techniques, the DR equation and DFT, Carbon 48 (2010) 2478-2486.

[6] F. Stoeckli, T.A. Centeno, Optimization of the characterization of porous carbons for supercapacitors, J. Mater. Chem. A 1 (2013) 6865-6873.

[7] M. Thommes, K. Kaneko, A.V. Neimark, J.P. Olivier, F. Rodriguez-Reinoso, J. Rouquerol, K.S.W. Sing, Physisorption of gases, with special reference to the evaluation of surface area and pore size distribution (IUPAC Technical Report), Pure Appl. Chem. 87 (2015) 10511069.

[8] G. Moreno-Fernandez, S. Perez-Ferreras, L. Pascual, I. Llorente, J. Ibañez, J.M. Rojo, Electrochemical study of tetraalkylammonium tetrafluoroborate electrolytes in combination with microporous and mesoporous carbon monoliths, Electrochim. Acta 268 (2018) 121-130.

[9] M.A. Moreno-Fernandez, Types of electrolytes for carbon-based supercapacitors. Ph. D. Thesis, Universidad Autónoma de Madrid, Madrid (19-December-2016) page 86.

[10] F. Stoeckli, in Porosity in carbons. Characterization and applications, ed. J. Patrick, Arnold, London, 1995, Ch. 3, 67-92.

[11] T.A. Centeno, J.A. Fernandez, F. Stoeckli, Correlation between heats of immersion and limiting capacitances in porous carbons, Carbon 46 (2008) 1025-1030.

[12] F. Stoeckli, T.A. Centeno, On the characterization of microporous carbons by immersion calorimetry alone, Carbon 35 (1997) 1097-1100. 


\section{Captions to Figure}

Figure 1. Pore size distribution (a) and cumulative surface area (b) obtained by different methods for the carbon monolith MicroCM. DFT combines $\mathrm{CO}_{2}$ (NLDFT) and $\mathrm{N}_{2}(\mathrm{QSDFT})$ analysis.
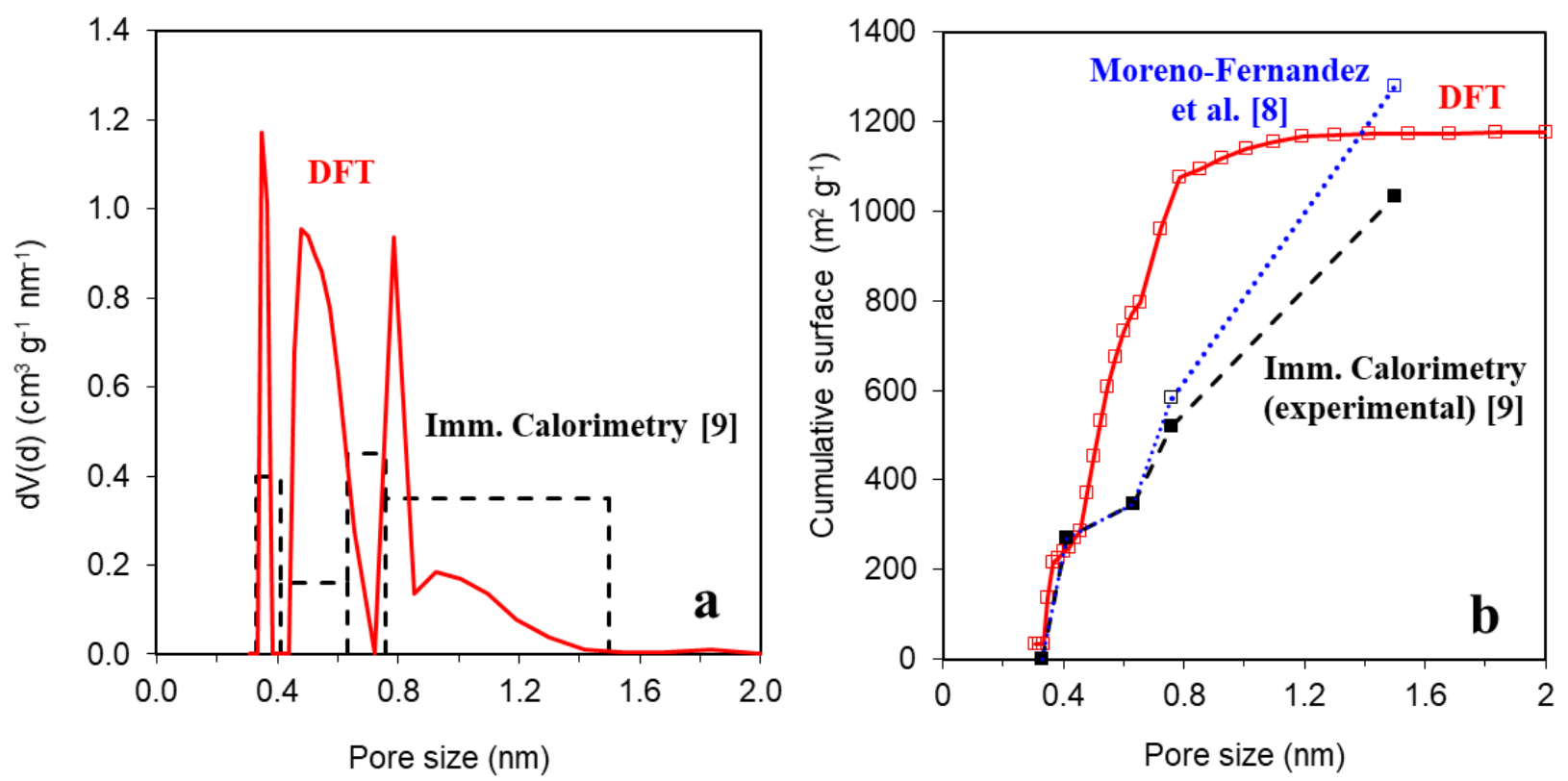


\section{Table 1}

Values of specific surface area $\left(\mathrm{m}^{2} \mathrm{~g}^{-1}\right)$ and their distribution in pores with different sizes assessed for MicroCM by diverse methods

\begin{tabular}{cccc}
\hline & $\begin{array}{c}\text { Moreno-Fernandez } \\
\text { et al. [8] }\end{array}$ & $\begin{array}{c}\text { Immersion } \\
\text { Calorimetry [9] }\end{array}$ & $\begin{array}{c}\text { DFT analysis of } \\
\mathrm{CO}_{2} \text { and } \mathrm{N}_{2} \text { adsorption }\end{array}$ \\
\cline { 2 - 4 } Total surface area & 1280 & 1033 & 1178 \\
$>0.33 \mathrm{~nm}$ & 1280 & 1033 & 1178 \\
$>0.41 \mathrm{~nm}$ & 1010 & 763 & 1178 \\
$>0.56 \mathrm{~nm}$ & {$[958]^{\mathrm{a}}$} & {$[710]^{\mathrm{a}}$} & 802 \\
$>0.63 \mathrm{~nm}$ & 933 & 686 & 356 \\
$>0.76 \mathrm{~nm}$ & 698 & 513 & 149 \\
$>0.90 \mathrm{~nm}$ & {$[560]^{\mathrm{a}}$} & {$[416]^{\mathrm{a}}$} & 70 \\
$>1.0 \mathrm{~nm}$ & {$[473]^{\mathrm{a}}$} & {$[347]^{\mathrm{a}}$} & 40 \\
\hline
\end{tabular}

${ }^{\mathrm{a}}$ [estimated by interpolation] 